\title{
A Generalized Interval LU Decomposition for the Solution of Interval Linear Systems
}

\author{
Alexandre Goldsztejn ${ }^{1}$ and Gilles Chabert ${ }^{2}$ \\ ${ }^{1}$ University of Central Arkansas, Conway 72035 Arkansas, USA \\ AGoldsztejn@uca.edu \\ ${ }^{2}$ Projet Coprin, INRIA, 2004 route des Lucioles, 06902 Sophia Antipolis, France \\ Gilles.Chabert@sophia.inria.fr
}

\begin{abstract}
Generalized intervals (intervals whose bounds are not constrained to be increasingly ordered) extend classical intervals providing better algebraic properties. In particular, the generalized interval arithmetic is a group for addition and for multiplication of zero free intervals. These properties allow one constructing a $\mathrm{LU}$ decomposition of a generalized interval matrix $\mathbf{A}$ : the two computed generalized interval matrices $\mathbf{L}$ and $\mathbf{U}$ satisfy $\mathbf{A}=\mathbf{L} \mathbf{U}$ with equality instead of the weaker inclusion obtained in the context of classical intervals. Some potential applications of this generalized interval LU decomposition are investigated.
\end{abstract}

\section{Introduction}

The LU decomposition of a square real matrix $A$ consists of computing two matrices $L$ and $U$ being respectively lower and upper triangular and satisfying $A=L U$. Such a decomposition eases the solution of many problems such as solving linear equations, inverting matrices, etc..

The Gauss elimination algorithm allows constructing LU decompositions. It has been generalized to interval matrices (see e.g. 1]). The interval Gauss elimination is widely used in order to construct an outer approximation of united solution sets, i.e. $\left\{x \in \mathbb{R}^{n} \mid(\exists A \in \mathbf{A})(\exists b \in \mathbf{b})(A x=b)\right\}$, where boldface letters mean interval matrices and vectors. In the study of the interval Gauss elimination, an interval LU decomposition has been introduced (cf. [1]). This interval LU decomposition does not satisfy $\mathbf{A}=\mathbf{L} \mathbf{U}$ but only the weaker relation $\mathbf{A} \subseteq \mathbf{L} \mathbf{U}$, which is well suited for outer approximation of united solution sets.

Generalized intervals are intervals whose bounds are not constrained to be increasingly ordered (e.g. $[-1,1]$ is a proper interval while $[1,-1]$ is an improper interval). They have been introduced in [23] in order to improve the mathematical structure of intervals. The generalized interval arithmetic (also called Kaucher arithmetic) is a group for addition and for multiplication of zero free generalized intervals. Thanks to the framework of generalized intervals, a new $\mathrm{LU}$ decomposition of a generalized interval matrix $\mathbf{A}$ is introduced. It satisfies $\mathbf{A}=\mathbf{L} \mathbf{U}$.

T. Boyanov et al. (Eds.): NMA 2006, LNCS 4310, pp. 312 319 2007.

(C) Springer-Verlag Berlin Heidelberg 2007 
The paper is organized as follows: Section 2 gives an overview of generalized intervals and their arithmetic. Section 3 presents the generalized interval LU decomposition, and Section 4 shows how it can be used to compute approximations of linear AE-solution sets.

\section{Generalized Intervals}

Generalized intervals are intervals whose bounds are not constrained to be ordered, for example $[-1,1]$ and $[1,-1]$ are generalized intervals. They have been introduced in [2] so as to improve the algebraic structure of intervals, while maintaining the inclusion monotonicity. The set of generalized intervals is denoted by $\mathbb{K} \mathbb{R}$ and is divided into three subsets:

- The set of proper intervals with bounds ordered increasingly. These proper intervals are identified with classical intervals. The set of proper intervals is denoted $\mathbb{I} R:=\{[a, b] \mid a \leq b\}$. Strictly proper intervals satisfy $a<b$.

- The set of improper intervals with bounds ordered decreasingly. It is denoted by $\overline{\mathbb{I R}}:=\{[a, b] \mid a \geq b\}$. Strictly proper intervals satisfy $a>b$.

- The set of degenerated intervals $\{[a, b] \mid a=b\}=\mathbb{I} \cap \overline{\mathbb{I R}}$. Degenerated intervals are identified to reals.

Therefore, from a set of reals $\{x \in \mathbb{R} \mid a \leq x \leq b\}$, one can build the two generalized intervals $[a, b]$ and $[b, a]$. It will be convenient to switch from one to the other keeping the underlying set of reals unchanged. To this purpose, the following three operations are introduced: the dual operation is defined by dual $[a, b]=[b, a]$; the proper projection is defined by pro $[a, b]=[\min \{a, b\}, \max$ $\{a, b\}]$; the improper projection is defined by $\operatorname{imp}[a, b]=[\max \{a, b\}, \min \{a, b\}]$.

The generalized intervals are partially ordered by an inclusion which extends the inclusion of classical intervals. Given two generalized intervals $\mathbf{x}=[\underline{\mathbf{x}}, \overline{\mathbf{x}}]$ and $\mathbf{y}=[\underline{\mathbf{y}}, \overline{\mathbf{y}}]$, the inclusion is defined by $\mathbf{x} \subseteq \mathbf{y} \Longleftrightarrow \underline{\mathbf{y}} \leq \underline{\mathbf{x}} \wedge \overline{\mathbf{x}} \leq \overline{\mathbf{y}}$. For example, $[-1, \overline{1]} \subseteq[-1.1,1.1]$ (this matches the set inclusion), $[1.1,-1.1] \subseteq[1,-1]$ (the inclusion between the underlying sets of real is reversed for improper intervals) and $[2,0.9] \subseteq[-1,1]$. As degenerated intervals are identified to reals, if $\mathbf{x}$ is proper then $x \in \mathbf{x} \Longleftrightarrow x \subseteq \mathbf{x}$. On the other hand, if $\mathbf{x}$ is strictly improper then for all $x \in \mathbb{R}$ the inclusion $x \subseteq \mathbf{x}$ is false.

The generalized interval arithmetic (also called Kaucher arithmetic) extends the classical interval arithmetic. Its definition can be found in [45]. When only proper intervals are involved, this arithmetic coincides with the interval arithmetic: $\mathbf{x} \circ \mathbf{y}=\{x \circ y \in \mathbb{R} \mid x \in \mathbf{x}, y \in \mathbf{y}\}$. When proper and improper intervals are involved, some new expressions are used. For example, $[a, b]+[c, d]=$ $[a+c, b+d]$ and if $a, b, c, d \geq 0$ then $[a, b] \times[c, d]=[a \times c, b \times d]$. The following useful property provides some bounds on the proper projection of the results of the generalized interval arithmetic. Let us consider $\mathbf{x}, \mathbf{y} \in \mathbb{K} \mathbb{R}$ and $\circ \in\{+,-, \times, /\}$. If $($ pro $\mathbf{x}) \circ($ pro $\mathbf{y})$ is defined then $\mathbf{x} \circ \mathbf{y}$ is defined and it satisfies

$$
\operatorname{pro}(\mathbf{x} \circ \mathbf{y}) \subseteq(\text { pro } \mathbf{x}) \circ(\text { pro } \mathbf{y})
$$


Generalized interval arithmetic has better algebraic properties than the classical interval arithmetic: the addition in $\mathbb{K} \mathbb{R}$ is a group. The opposite of an interval $\mathbf{x}$ is - dual $\mathbf{x}$, i.e.,

$$
\mathbf{x}+(- \text { dual } \mathbf{x})=\mathbf{x}-\text { dual } \mathbf{x}=[0,0]
$$

The multiplication in $\mathbb{K} \mathbb{R}$ restricted to generalized intervals whose proper projection does not contain 0 is also a group. The inverse of such a generalized interval $\mathbf{x}$ is $1 /($ dual $\mathbf{x})$, i.e.,

$$
\mathbf{x} \times(1 / \text { dual } \mathbf{x})=\mathbf{x} /(\text { dual } \mathbf{x})=[1,1] .
$$

Although addition and multiplication in $\mathbb{K} \mathbb{R}$ are associative, they are not distributive. The addition and multiplication in $\mathbb{K} \mathbb{R}$ are linked by the following distributivity laws (see 667/5]). Whatever are $\mathbf{x}, \mathbf{y}, \mathbf{z} \in \mathbb{K} \mathbb{R}$,

- conditional distributivity:

$$
\mathbf{x} \times \mathbf{y}+(\operatorname{imp} \mathbf{x}) \times \mathbf{z} \subseteq \mathbf{x} \times(\mathbf{y}+\mathbf{z}) \subseteq \mathbf{x} \times \mathbf{y}+(\text { pro } \mathbf{x}) \times \mathbf{z} .
$$

The three following particular cases will be of practical interest in this paper.

- subdistributivity: if $\mathbf{x} \in \mathbb{I} R$ then $\mathbf{x} \times(\mathbf{y}+\mathbf{z}) \subseteq \mathbf{x} \times \mathbf{y}+\mathbf{x} \times \mathbf{z}$;

- superdistributivity: if $\mathbf{x} \in \overline{\mathbb{I R}}$ then $\mathbf{x} \times(\mathbf{y}+\mathbf{z}) \supseteq \mathbf{x} \times \mathbf{y}+\mathbf{x} \times \mathbf{z}$;

- distributivity: if $x \in \mathbb{R}$ then $x \times(\mathbf{y}+\mathbf{z})=x \times \mathbf{y}+x \times \mathbf{z}$.

Another useful property of the Kaucher arithmetic is its monotonicity with respect to the inclusion: whatever are $\circ \in\{+, \times,-, \div\}$ and $\mathbf{x}, \mathbf{y}, \mathbf{x}^{\prime}, \mathbf{y}^{\prime} \in \mathbb{K} \mathbb{R}$,

$$
\mathbf{x} \subseteq \mathbf{x}^{\prime} \wedge \mathbf{y} \subseteq \mathbf{y}^{\prime} \Longrightarrow(\mathbf{x} \circ \mathbf{y}) \subseteq\left(\mathbf{x}^{\prime} \circ \mathbf{y}^{\prime}\right) .
$$

The next example illustrates the way these properties will be used in the sequel.

Example 1. Consider the expression $\mathbf{x}+\mathbf{u v} \subseteq \mathbf{y}$. Subtracting dual $(\mathbf{u v})=$ (dual $\mathbf{u})($ dual $\mathbf{v})$ to each side preserves the inclusion: $\mathbf{x}+\mathbf{u v}-$ dual $(\mathbf{u v}) \subseteq$ $\mathbf{y}-($ dual $\mathbf{u})($ dual $\mathbf{v})$. As - dual (uv) is the opposite of $\mathbf{u v}$, the following inclusion is eventually proved to hold: $\mathbf{x} \subseteq \mathbf{y}-($ dual $\mathbf{u})($ dual $\mathbf{v})$.

Finally, generalized interval vectors $\mathbf{x} \in \mathbb{K}^{n}$ and generalized interval matrices $\mathbf{A} \in \mathbb{K}^{n \times n}$ together with their additions and multiplications are defined similarly to their real and classical interval counterparts.

\section{Generalized Interval LU Decomposition}

A Gauss elimination algorithm for generalized interval matrices is first presented. It will be used to construct the generalized interval LU decomposition. Consider a generalized interval matrix $\mathbf{A}=\left(\mathbf{A}_{i j}\right)$. In order to obtain a generalized interval LU decomposition, the Gauss elimination algorithm is applied in the following 
way: if $0 \notin$ pro $\mathbf{A}_{11}$, for $i \in[2 . . n]$ multiply the first row by $\mathbf{A}_{i 1} /\left(\right.$ dual $\left.\mathbf{A}_{11}\right)$. As $\mathbf{A}_{11} /\left(\right.$ dual $\left.\mathbf{A}_{11}\right)=1$ the following row is obtained:

$$
\left(\mathbf{A}_{i 1}, \frac{\mathbf{A}_{12} \mathbf{A}_{i 1}}{\text { dual } \mathbf{A}_{11}}, \cdots, \frac{\mathbf{A}_{1 n} \mathbf{A}_{i 1}}{\text { dual } \mathbf{A}_{11}}\right) .
$$

The second step consists in subtracting the dual of the previously computed row to the $i^{t h}$ row of the matrix $\mathbf{A}$. As $\mathbf{A}_{i 1}-$ dual $\mathbf{A}_{i 1}=0$ one obtains

$$
\left(0, \mathbf{A}_{i 2}-\frac{\left(\text { dual } \mathbf{A}_{12}\right)\left(\text { dual } \mathbf{A}_{i 1}\right)}{\mathbf{A}_{11}}, \cdots, \mathbf{A}_{i n}-\frac{\left(\text { dual } \mathbf{A}_{1 n}\right)\left(\text { dual } \mathbf{A}_{i 1}\right)}{\mathbf{A}_{11}}\right) .
$$

Once this transformation is applied for each $i \in[2 . . n]$, the following interval matrix $\mathbf{A}$ is obtained:

$$
\mathbf{A}:=\left(\begin{array}{cccc}
\mathbf{A}_{11} & \mathbf{A}_{12} & \cdots & \mathbf{A}_{1 n} \\
0 & \mathbf{A}_{22}^{\prime} & \cdots & \mathbf{A}_{2 n}^{\prime} \\
\vdots & \vdots & \ddots & \vdots \\
0 & \mathbf{A}_{n 2}^{\prime} & \cdots & \mathbf{A}_{n n}^{\prime}
\end{array}\right) \text {, where } \mathbf{A}_{i j}^{\prime}:=\mathbf{A}_{i j}-\frac{\left(\text { dual } \mathbf{A}_{1 j}\left(\text { dual } \mathbf{A}_{i 1}\right)\right.}{\mathbf{A}_{11}} .
$$

As in the context of real numbers and classical intervals, this leads to a $\mathrm{LU}$ decomposition of the generalized interval matrix A. This LU decomposition can be formulated in the following way:

$$
\begin{gathered}
\mathbf{L}_{i i}=1 \text { and } \mathbf{L}_{i j}=0 \text { for } i<j, \\
\mathbf{L}_{i j}=\left(\mathbf{A}_{i j}-\sum_{k<j} \operatorname{dual}\left(\mathbf{L}_{i k} \mathbf{U}_{k j}\right)\right) /\left(\operatorname{dual} \mathbf{U}_{i i}\right) \quad \text { for } j<i, \\
\mathbf{U}_{i j}=0 \text { for } i>j, \\
\mathbf{U}_{i j}=\mathbf{A}_{i j}-\sum_{k<i} \operatorname{dual}\left(\mathbf{L}_{i k} \mathbf{U}_{k j}\right) \quad \text { for } i \leq j .
\end{gathered}
$$

The previous expressions allow constructing a generalized interval LU decomposition of $\mathbf{A}$ : the recursive construction is started by the first row of $\mathbf{U}$ which is trivially computed using (12). It is equal to the first row of $\mathbf{A}$. Then, provided that $0 \notin$ pro $\mathbf{U}_{i i}$, the $i^{\text {th }}$ column of $\mathbf{L}$ using (10) and the $i^{\text {th }}$ row of $\mathbf{U}$ is constructed using (12). This process is recursively repeated for $i+1$.

Proposition 1. Let $\mathbf{A} \in \mathbb{K}^{n \times n}$ be a generalized interval matrix. Provided that the generalized interval matrices $\mathbf{L}$ and $\mathbf{U}$ defined by (9-12) can be constructed, they satisfy $\mathbf{A}=\mathbf{L} \mathbf{U}$.

Proof. Consider $i, j \in[1 . . n]$ such that $i \leq j$. Then by equation (12)

$$
\mathbf{U}_{i j}=\mathbf{A}_{i j}-\sum_{k<i} \operatorname{dual}\left(\mathbf{L}_{i k} \mathbf{U}_{k j}\right) .
$$


Adding $\sum_{k<i} \mathbf{L}_{i k} \mathbf{U}_{k j}$ to each side of the equality, $\mathbf{U}_{i j}+\sum_{k<i} \mathbf{L}_{i k} \mathbf{U}_{k j}=\mathbf{A}_{i j}$. As $\mathbf{L}_{j j}=1$ and $\mathbf{L}_{i k}=0$ for $i<k, \sum_{k \in[1 . . n]} \mathbf{L}_{i k} \mathbf{U}_{k j}=\mathbf{A}_{i j}$. Considering the equation (10), we argue similarly in the case $i, j \in[1 . . n]$, with $i>j$.

Two examples of LU decomposition are now presented.

Example 2. Consider the interval matrix

$$
\mathbf{A}=\left(\begin{array}{ccc}
{[9,11]} & {[-1,1]} & {[-1,1]} \\
{[-11,11]} & {[8,12]} & {[-2,2]} \\
{[-11,11]} & {[-12,12]} & {[7,13]}
\end{array}\right)
$$

The computations of the generalized interval LU decomposition are detailed: the first row of $\mathbf{U}$ is the first row of $\mathbf{A}$. Now, using (10) and (12),

$$
\begin{aligned}
\mathbf{L}_{21} & =\mathbf{A}_{21} /\left(\operatorname{dual} \mathbf{U}_{11}\right)=[-11,11] /[11,9]=[-1,1], \\
\mathbf{L}_{31} & =\mathbf{A}_{31} /\left(\operatorname{dual} \mathbf{U}_{11}\right)=[-11,11] /[11,9]=[-1,1], \\
\mathbf{U}_{22} & =\mathbf{A}_{22}-\operatorname{dual}\left(\mathbf{L}_{21} \mathbf{U}_{12}\right)=[9,11] \\
\mathbf{U}_{23} & =\mathbf{A}_{23}-\operatorname{dual}\left(\mathbf{L}_{21} \mathbf{U}_{13}\right)=[-1,1], \\
\mathbf{L}_{32} & =\left(\mathbf{A}_{32}-\operatorname{dual}\left(\mathbf{L}_{31} \mathbf{U}_{12}\right)\right) /\left(\operatorname{dual} \mathbf{U}_{22}\right)=[-1,1], \\
\mathbf{U}_{33} & =\mathbf{A}_{33}-\operatorname{dual}\left(\mathbf{L}_{31} \mathbf{U}_{13}\right)-\operatorname{dual}\left(\mathbf{L}_{32} \mathbf{U}_{23}\right)=[9,11]
\end{aligned}
$$

Therefore, we obtain following interval matrices:

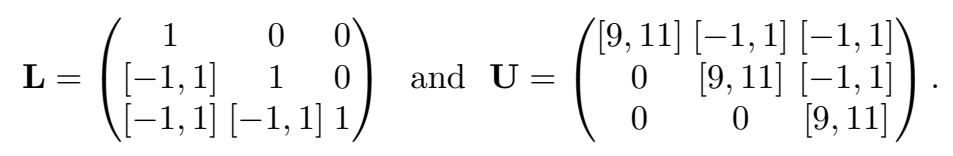

These interval matrices satisfy $\mathbf{A}=\mathbf{L} \mathbf{U}$.

Example 3. Consider the interval matrix

$$
\mathbf{A}=\left(\begin{array}{ccc}
{[2,3]} & 1 & 0 \\
1 & {[2,3]} & 1 \\
0 & 1 & {[2,3]}
\end{array}\right)
$$

The following interval matrices correspond to the generalized interval LU decomposition of $\mathbf{A}$ :

$$
\mathbf{L}=\left(\begin{array}{ccc}
1 & 0 & 0 \\
{\left[0.5, \frac{1}{3}\right]} & 1 & 0 \\
0 & {\left[\frac{2}{3}, 0.375\right]} & 1
\end{array}\right) \quad \text { and } \mathbf{U}=\left(\begin{array}{ccc}
{[2,3]} & 1 & 0 \\
0 & {\left[1.5, \frac{8}{3}\right]} & 1 \\
0 & 0 & {\left[\frac{4}{3}, 2.625\right]}
\end{array}\right)
$$

These interval matrices satisfy $\mathbf{A}=\mathbf{L} \mathbf{U}$. Notice that $\mathbf{L}$ is not proper anymore.

As a direct consequence of the property (1), given a generalized interval matrix $\mathbf{A} \in \mathbb{K}^{n \times n}$, if (pro A) has a classical interval LU decomposition (as defined in [1]) then $\mathbf{A}$ has a generalized interval LU decomposition. 


\section{Approximation of Linear AE-Solution Set}

We define linear AE-solution sets using a different convention than the one proposed in [5]. The justification of this new convention is out of the scope of this paper (cf. 8]). Let $\mathbf{A} \in \mathbb{K}^{n \times n}$ be a generalized interval matrix, and $\mathbf{b} \in \mathbb{K}^{n}$ be a generalized interval vector. Define $\mathbf{A}^{\forall}, \mathbf{A}^{\exists} \in \mathbb{I} \mathbb{R}^{n \times n}$ and $\mathbf{b}^{\forall}, \mathbf{b}^{\exists} \in \mathbb{I} \mathbb{R}^{n}$ by

$$
\begin{array}{ll}
\mathbf{A}_{i j}^{\exists}:= \begin{cases}\mathbf{A}_{i j} \text { if } \mathbf{A}_{i j} \in \mathbb{R}, \\
0 \text { otherwise. }\end{cases} & \mathbf{A}_{i j}^{\forall}:= \begin{cases}\text { pro } \mathbf{A}_{i j} \text { if } \mathbf{A}_{i j} \in \overline{\mathbb{R}}, \\
0 & \text { otherwise. }\end{cases} \\
\mathbf{b}_{i}^{\exists}:= \begin{cases}\mathbf{b}_{i} \text { if } \mathbf{b}_{i} \in \mathbb{I R}, \\
0 \text { otherwise. }\end{cases} & \mathbf{b}_{i}^{\forall}:= \begin{cases}\text { pro } \mathbf{b}_{i} \text { if } \mathbf{b}_{i} \in \overline{\mathbb{R}}, \\
0 & \text { otherwise. }\end{cases}
\end{array}
$$

So $\mathbf{A}=\mathbf{A}^{\exists}+\left(\right.$ dual $\left.\mathbf{A}^{\forall}\right)$ and $\mathbf{b}=\mathbf{b}^{\exists}+\left(\right.$ dual $\left.\mathbf{b}^{\forall}\right)$. Then, the linear AE-solution set $\Sigma(\mathbf{A}, \mathbf{b})$ is the following subset of $\mathbb{R}^{n}$ :

$$
\left(\forall A^{\forall} \in \mathbf{A}^{\forall}\right)\left(\forall b^{\forall} \in \mathbf{b}^{\forall}\right)\left(\exists A^{\exists} \in \mathbf{A}^{\exists}\right)\left(\exists b^{\exists} \in \mathbf{b}^{\exists}\right)\left(A^{\forall}+A^{\exists}\right) x=\left(b^{\forall}+b^{\exists}\right) .
$$

We can exhibit the following two special cases: if $\mathbf{A}$ and $\mathbf{b}$ are proper, then $\Sigma(\mathbf{A}, \mathbf{b})=\left\{x \in \mathbb{R}^{n} \mid \exists A \in \mathbf{A}, \exists b \in \mathbf{b}, A x=b\right\}$, which is called a united solution set. While, if $\mathbf{A}$ is improper and $\mathbf{b}$ is proper, then $\Sigma(\mathbf{A}, \mathbf{b})=\left\{x \in \mathbb{R}^{n} \mid \forall A \in\right.$ pro $\mathbf{A}, \exists b \in \mathbf{b}, A x=b\}$, which is called a tolerable solution set. Shary has discovered a very useful characterization of linear AE-solution sets (cf. 5. Theorem 5.1, p. 375]). Let us reproduce his proof with our new conventions. (24) can be written $\left(\forall A^{\forall} \in \mathbf{A}^{\forall}\right)\left(\forall b^{\forall} \in \mathbf{b}^{\forall}\right)\left(\exists A^{\exists} \in \mathbf{A}^{\exists}\right)\left(\exists b^{\exists} \in \mathbf{b}^{\exists}\right) A^{\forall} x-b^{\forall}=b^{\exists}-A^{\exists} x$. Now as every quantified parameter has only one occurrence in the expression, interval arithmetic leads to the exact range and we obtain the following equivalent condition: $\mathbf{A}^{\forall} x-\mathbf{b}^{\forall} \subseteq \mathbf{b}^{\exists}-\mathbf{A}^{\exists} x$. Now, using the group property of the Kaucher arithmetic, the previous condition is equivalent to $\mathbf{A}^{\forall} x+\operatorname{dual}\left(\mathbf{A}^{\exists} x\right) \subseteq \mathbf{b}^{\exists}+\operatorname{dual} \mathbf{b}^{\forall}$. Finally, notice that dual $\left(\mathbf{A}^{\exists} x\right)=\left(\right.$ dual $\left.\mathbf{A}^{\exists}\right) x$ and use the distributivity w.r.t. $x$ to obtain

$$
x \in \Sigma(\mathbf{A}, \mathbf{b}) \Longleftrightarrow(\text { dual } \mathbf{A}) x \subseteq \mathbf{b} .
$$

This characterization only differs from [5, Theorem 5.1, p. 375] by the new conventions we use. It can be used in order to obtain generalized interval operators that help to approximate linear AE-solution sets. In particular, outer approximations can be done using the generalized interval Gauss-Seidel iteration introduced in 9,5]. The generalized interval LU decomposition is now used to build inner and outer approximations of linear AE-solution sets $\Sigma(\mathbf{A}, \mathbf{b})$.

Theorem 1. Let $\mathbf{A} \in \mathbb{K}^{n \times n}, \mathbf{b} \in \mathbb{K}^{n}$. Let $\mathbf{L}$ and $\mathbf{U}$ be the generalized interval $L U$ decomposition of $\mathbf{A}$. Then define the generalized interval vectors $\mathbf{x}, \mathbf{x}^{\prime}, \mathbf{y}, \mathbf{y}^{\prime} \in$ $\mathbb{K R}^{n}$ such that for all $i \in[1 . . n]$

$$
\begin{gathered}
\mathbf{y}_{i}=\mathbf{b}_{i}-\sum_{j<i} \mathbf{L}_{i j}\left(\text { dual } \mathbf{y}_{j}\right) \text { and } \mathbf{x}_{i}=\left(\mathbf{y}_{i}-\sum_{j>i} \mathbf{U}_{i j}\left(\text { dual } \mathbf{x}_{j}\right)\right) / \mathbf{U}_{i i} . \\
\mathbf{y}_{i}^{\prime}=\mathbf{b}_{i}-\sum_{j<i} \mathbf{L}_{i j} \mathbf{y}_{j}^{\prime} \text { and } \mathbf{x}_{i}^{\prime}=\left(\mathbf{y}_{i}^{\prime}-\sum_{j>i} \mathbf{U}_{i j} \mathbf{x}_{j}^{\prime}\right) / \mathbf{U}_{i i}
\end{gathered}
$$




\section{Both following properties hold:}

(i) If $\mathbf{L}$ is proper and $\mathbf{x}$ is proper then $\mathbf{x} \subseteq \Sigma(\mathbf{A}, \mathbf{b})$.

(ii) Suppose that $\mathbf{U}$ and $\mathbf{L}$ are improper. If $\mathbf{x}^{\prime}$ is proper then $\Sigma(\mathbf{A}, \mathbf{b}) \subseteq \mathbf{x}^{\prime}$. Otherwise, $\Sigma(\mathbf{A}, \mathbf{b})=\emptyset$.

Proof. As a direct consequence of the construction of the LU decomposition, if $\mathbf{A}=\mathbf{L} \mathbf{U}$ then $($ dual $\mathbf{A})=($ dual $\mathbf{L})($ dual $\mathbf{U})$.

(i) By definition of $\mathbf{y}$ and $\mathbf{x}$ we have (dual $\mathbf{L}) \mathbf{y}=\mathbf{b}$ and (dual $\mathbf{U}) \mathbf{x}=\mathbf{y}$. Therefore we obtain (dual $\mathbf{L})(($ dual $\mathbf{U}) \mathbf{x})=\mathbf{b}$. As $\mathbf{L}$ is supposed to be proper, (dual $\mathbf{L}$ ) is improper. Consider any $x \in \mathbf{x}$. A componentwise application of the super-distributivity shows $(($ dual $\mathbf{L})($ dual $\mathbf{U})) x \subseteq($ dual $\mathbf{L})(($ dual $\mathbf{U}) x)$. That is (dual $\mathbf{A}) x \subseteq \mathbf{b}$ and finally $x \in \Sigma(\mathbf{A}, \mathbf{b})$ thanks to (25).

(ii) Assume $x \in \Sigma(\mathbf{A}, \mathbf{b})$. Using (25) we obtain ((dual L)(dual $\mathbf{U})) x \subseteq \mathbf{b}$. As both (dual $\mathbf{L}$ ) and (dual $\mathbf{U}$ ) are proper, $\mathbf{b}$ has to be proper. Also, the subdistributivity law proves (dual L) ((dual U) $x) \subseteq(($ dual $\mathbf{L})($ dual $\mathbf{U})) x$, so (dual $\mathbf{L})(($ dual $\mathbf{U}) x) \subseteq \mathbf{b}$. As (dual $\mathbf{U})$ is proper, $\mathbf{u}:=($ dual $\mathbf{U}) x$ is proper too. Claim: $\mathbf{u} \subseteq \mathbf{y}^{\prime}$. We shall prove it by induction. The first line of (dual $\left.\mathbf{L}\right) \mathbf{u} \subseteq \mathbf{b}$ yields $\mathbf{u}_{1} \subseteq \mathbf{b}_{1}$ while $\mathbf{b}_{1}=\mathbf{y}_{1}^{\prime}$ by definition of $\mathbf{y}^{\prime}$. Now fix $i \in\{2, \ldots, n-$ $1\}$ and suppose $j<i \Rightarrow \mathbf{u}_{j} \subseteq \mathbf{y}_{j}^{\prime}$. The $i^{\text {th }}$ line of (dual $\left.\mathbf{L}\right) \mathbf{u} \subseteq \mathbf{b}$ yields $\mathbf{u}_{i}+\sum_{j<i}\left(\right.$ dual $\left.\mathbf{L}_{i j}\right) \mathbf{u}_{j} \subseteq \mathbf{b}_{i}$. Therefore $\mathbf{u}_{i} \subseteq \mathbf{b}_{i}-\sum_{j<i} \mathbf{L}_{i j}\left(\right.$ dual $\left.\mathbf{u}_{j}\right) \subseteq \mathbf{b}_{i}-$ $\sum_{j<i} \mathbf{L}_{i j} \mathbf{u}_{j}$, the second inclusion being a consequence of inclusion monotonicity (dual $\mathbf{u}_{j} \subseteq \mathbf{u}_{j}$ because $\mathbf{u}_{j}$ is proper). Finally, using the induction hypothesis and inclusion monotonicity, we obtain $\mathbf{u}_{i} \subseteq \mathbf{b}_{i}-\sum_{j<i} \mathbf{L}_{i j} \mathbf{y}_{j}^{\prime}$ which is equal to $\mathbf{y}_{i}^{\prime}$ by definition. So far, we have proved (dual $\mathbf{U}) x \subseteq \mathbf{y}^{\prime}$. Using induction in a similar way as previously, on can prove $x \subseteq \mathrm{x}^{\prime}$. As a consequence, if $\mathrm{x}^{\prime}$ is proper then $\Sigma(\mathbf{A}, \mathbf{b}) \subseteq \mathbf{x}^{\prime}$. Otherwise $\Sigma(\mathbf{A}, \mathbf{b})=\emptyset$.

Due to the conditions required for the LU decomposition in Theorem 1, outer approximation is likely to succeed when $\mathbf{A}$ is improper, i.e. when a tolerable solution set is under consideration. While, inner approximation is likely to succeed when $\mathbf{A}$ is proper, i.e. when a united solution set is under consideration. Under these conditions, experimentations have shown that the generalized interval LU decomposition applied to diagonally dominant matrices centered around the identity satisfies the conditions of Theorem 1. However, the generalized interval Gauss-Seidel operator and the direct resolution of the equation (dual $\mathbf{A}) \mathbf{x}=\mathbf{b}$ seem to provide close but better results for this class of matrices. It still remains that in presence of several solution sets sharing the same interval matrix, the generalized interval LU decomposition needs to be computed only once, thereby sparing computation time.

In some situations, the generalized interval LU decomposition can provide a much sharper outer approximation than the generalized interval Gauss-Seidel (GIGS) operator. This is illustrated in the next example.

Example 4. Consider the tridiagonal matrix $\mathbf{A}$ and vector $\mathbf{b}$ defined by

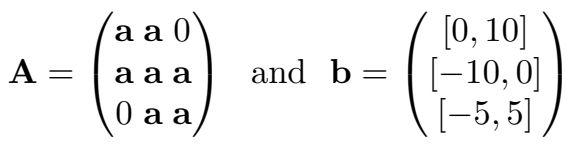


with $\mathbf{a}=[1.1,0.9]$ and the tolerable solution set $\Sigma(\mathbf{A}, \mathbf{b})$. Both the GIGS and the generalized interval LU decomposition have a bad behavior on this example. However, exchanging raws 2 and 3 leads to better behavior. Starting from the initial box $( \pm 1000, \pm 1000, \pm 1000)^{\top}$ the GIGS operator gives raise to $([-18.4091,27.5],[-22.5,22.5],[-27.5,18.4091])^{\top}$. The generalized interval LU decomposition raises the following outer approximation:

$$
([-12.8099,11.157],[-9.12847,19.5718],[-13.6364,4.54545])^{\top},
$$

which is much sharper than the one computed by the GIGS operator.

It must be noted that changing slightly the uncertainties in the interval matrix of the previous example leads to a matrix $\mathbf{L}$ which is not proper any more. While this good result is promising, some additional developments will be necessary to deal with more general situations.

\section{Conclusion}

An interval LU decomposition has been proposed in the framework of generalized intervals. Thanks to the group structure of the generalized interval arithmetic, this new generalized interval LU decomposition satisfies $\mathbf{A}=\mathbf{L U}$. In addition to the theoretical interest of such a generalized interval LU decomposition, we proved that it can be used to construct some approximations of linear AE-solution sets. On some examples, these approximations may be much more accurate than the one computed by the generalized interval Gauss-Seidel operator. However, some stringent conditions about the proper/improper qualities of $\mathbf{L}$ and $\mathbf{U}$ restrict the application of the decomposition. Some work still have to be conducted to generalize these particular application cases.

\section{References}

1. Neumaier, A.: Interval Methods for Systems of Equations. Cambridge Univ. Press, Cambridge (1990)

2. Ortolf, H.J.: Eine Verallgemeinerung der Intervallarithmetik. Geselschaft fuer Mathematik und Datenverarbeitung, Bonn 11 (1969) 1-71

3. Kaucher, E.: Uber metrische und algebraische Eigenschaften einiger beim numerischen Rechnen auftretender Raume. PhD thesis, Karlsruhe (1973)

4. Kaucher, E.: Interval Analysis in the Extended Interval Space $\mathbb{I R}$. Computing, Suppl. 2 (1980) 33-49

5. Shary, S.: A new technique in systems analysis under interval uncertainty and ambiguity. Reliable computing 8 (2002) 321-418

6. Popova, E.: Multiplication distributivity of proper and improper intervals. Reliable computing $\mathbf{7}(2)$ (2001) 129-140

7. SIGLA/X group: Modal intervals. Reliab. Comp. 7 (2001) 77-111

8. Goldsztejn, A., Chabert, G.: On the approximation of linear AE-solution sets. In: 12th GAMM IMACS International Symposion on Scientific Computing, Computer Arithmetic and Validated Numerics, Duisburg, Germany (2006)

9. Shary, S.: Interval Gauss-Seidel Method for Generalized Solution Sets to Interval Linear Systems. Reliable computing 7 (2001) 141-155 